\title{
Position of Transversal Competencies in University Settings
}

\section{[La place des competences transversales dans le milieu universitaire]}

\author{
Iveta Rizekova
}

\section{DOI: 10.18355/XL.2015.08.04.31-45}

\begin{abstract}
Résumé
Le présent article porte sur la problématique des compétences transversales en traçant des modalités de leur développement et leur application dans le milieu universitaire. Les compétences transversales constituent un référentiel pour l'épanouissement des capacités universelles de l'apprenti, telles que résoudre des problèmes, monter des projets ou encore exploiter des méthodes efficaces d'apprentissage. Des compétences acquises au cours de l'enseignement des langues ou des matières de spécialité en langues aident l'étudiant à réussir tant au niveau de communication en langues, qu'en diverses disciplines préparant aux activités de la vie professionnelle et personnelle. La présente communication vise les compétences transversales susceptibles d'éveiller l'intérêt des enseignants (des langues) dans les établissements d'enseignement supérieur.
\end{abstract}

Mots-clés

compétence, transversalité, langues, application, enseignement universitaire

\section{Introduction}

L'univers multidimentionnel dans lequel nous vivons nous oblige à exploiter diverses connaissances et compétences pour que nous nous adaptions le mieux aux conditions de vie changeantes. Il en découle la tâche pour les pédagogues du supérieur, contribuant largement à la formation des jeunes, de veiller au développement des connaissances génériques dont les diplômés pourront se servir dans des situations professionnelles et personnelles.

Aujourd'hui, tous les pays participant au processus de Bologne, visent à introduire un système académique comparable, à promouvoir la mobilité des étudiants, des enseignants et des chercheurs, à assurer la qualité de l'enseignement et à intégrer la dimension européenne dans l'enseignement supérieur. Ce processus implique l'idée, promotrice des pays signataires de la précédente déclaration de la Sorbonne datant de 1998, que « l'Europe que nous bâtissons n'est pas seulement celle de l'euro, des banques et de l'économie; elle doit être aussi une Europe du savoir» (Synthèse de la législation de l'UE). Certains pays européens, avaient avancé ces actes, s'étant aperçus que la réforme des systèmes d'enseignement était inéluctable, car les savoirs traditionnels ne s'avéraient plus suffisants pour répondre aux exigences de la société moderne. L'identification des compétences plus complexes arrive donc à l'ordre du jour. Par conséquent, la nouvelle approche par compétences traverse les débats contemporains dans différentes sphères, y compris évidemment, le milieu universitaire. Bien que les enseignants profitent d'une certaine liberté d'action sur le plan pédagogique, didactique et méthodologique, néanmoins, des programmes d'études et des syllabus des cours déterminent les référentiels des compétences demandées. L'objectif primordial est de concevoir et d'adapter des cours de langues (ou des matières en langues) aux besoins de la vie professionnelle et ceux du marché de travail dans la société contemporaine.

Dès le début de notre siècle, la réflexion sur les compétences, dites transversales, s'entend dans l'espace européen. Ces compétences attirent l'attention des enseignants ainsi que des chercheurs. Certes, il est plus aisé d'imposer la présence 
des compétences transversales dans le système éducatif que d'en assurer la mise en valeur réelle, étant donné la complexité de la transversalité. "Les compétences transversales constituent non seulement les démarches fondamentales de la pensée, transférables d'une matière à l'autre mais englobent également toutes les interactions sociales, cognitives, affectives, culturelles et psychomotrices entre l'apprenant et la réalité qui l'entoure ». L'enseignement universitaire devrait donc mettre l'apprenant dans des situations qui mobilisent ses savoirs, savoir-faire, savoir-être et savoirapprendre.

\section{Compétence du point de vue de diverses théories}

1.1 Points de repère sur la compétence

La compétence, ayant un caractère complexe, peut être définie de différentes façons. Mais la notion de la compétence est parfois confondue avec celle de capacités. Selon Barbier (César-Pirard, $2012: 15)$ les compétences représentent davantage des ressources mobilisables, développées dans l'espace-temps de formation et supposées être transférées dans l'espace-temps de production. Or les capacités sont à comprendre "comme des propriétés attribuées à des sujets dans leurs rapports avec des classes d'activités, construites par abstraction et dégagement d'invariants et considérées comme un potentiel pour ces mêmes sujets » (ibidem). En d'autres termes, les compétences aident à appliquer des connaissances, des savoir-faire et des attitudes personnels dans des situations de travail variées.

Dans ce contexte, la compétence est liée à la responsabilité et l'autonomie. Florence Legendre, sociologue française et chercheure au Centre universitaire de recherche en sciences de l'éducation de Picardie souligne l'idée que la notion de compétence s'est d'abord développée dans le contexte professionnel et dans le monde des entreprises. Dans les années $70 \mathrm{du} \mathrm{XX}^{\mathrm{e}}$ siècle, la notion de compétence commence progressivement à se substituer à celle de qualification qui englobe des savoir-faire et des techniques reconnus et sanctionnés par un diplôme. Dans le contexte professionnel, la compétence signifie une mise à l'épreuve de la responsabilité du salarié à l'égard du résultat. Elle requiert une certaine autonomie pour faire face à des situations non prévisibles (De la transmission des savoirs).

Le psychologue Jacques Leplat (Afriat, 2006 : 22) explique le concept de la compétence par certains traits caractéristiques: a) la compétence est indissociable des activités par lesquelles elle se manifeste; b) la compétence est opératoire, car elle n'a de sens que par rapport à l'action; c) le concept de la compétence doit toujours être précisé, ce qui veut dire que l'on est compétent pour une tâche ou un ensemble de tâches et que la compétence est relative à la situation concrète; d) la compétence est apprise, car c'est grâce à l'apprentissage théorique et à l'expérience que l'individu devient compétent; e) la compétence est structurée, comme elle combine les savoirs et les savoir-faire pour répondre à des exigences d'adaptation; e) la compétence est abstraite et hypothétique et par suite inobservable, étant donné que seulement les comportements de l'individu sont observables.

Le sociologue Philippe Zarifian définit la compétence comme «l'intelligence pratique des situations qui se manifeste par trois types de comportement: l'autonomie, la prise de responsabilité et la communication » (Afriat, 2006 : 22). Il est nécessaire alors que l'individu sache mobiliser d'une part, ses ressources personnelles, c'est-à-dire des savoirs théoriques, savoir-faire opérationnel et savoir-faire social; et d'autre part, des ressources extérieures, comme outils de travail, documentations, réseaux relationnels (ibid.).

Le professeur canadien Jacques Tardif considérait la compétence comme « un savoir-agir complexe prenant appui sur la mobilisation et la combinaison efficaces d'une variété de ressources internes et externes à l'intérieur d'une famille 
de situations » (Houchot - Robin, 2007 : 12). Marc Romainville, professeur belge, estime que " une compétence est un ensemble intégré et fonctionnel de savoirs, savoir-faire, savoir-être et savoir-devenir, qui permettront, face à une catégorie de situations, de s'adapter, de résoudre des problèmes et de réaliser des projets » (ibid.).

F. Legendre distingue deux pôles dans la notion de compétence: « une logique de mise en œuvre et une logique de réflexion. Le savoir-agir de la compétence suppose la capacité à investir dans l'action les savoirs précédemment acquis mais aussi la capacité à abstraire de ses actions des savoirs qui pourront être réinvestis dans de nouveaux contextes» (ibid.). Pour l'auteure «la contextualisation des connaissances constitue une première étape du processus de construction cognitive, leur décontextualisation en constitue la seconde: diversification des contextes d'apprentissage ou des problèmes, conceptualisation et intégration de tout concept nouveau dans un réseau, stimulation de la réflexion métacognitive » (ibid.).

Ces quelques exemples montrent la complexité de la notion de compétence, et donc la difficulté de la cerner. Malgré une certaine ambiguïté des définitions des termes, la diversité d'attitudes paraît être enrichissante, tant au niveau de la recherche que de la pratique. On observe diverses tentatives pour intégrer cette préoccupation dans le processus pédagogique dans les établissements scolaires.

\subsection{Notion de compétences transversales}

Les compétences dites transversales constituent un référentiel pour le développement des capacités générales, applicables à plusieurs contextes ou disciplines. Des compétences transversales ont le caractère formatif, c'est-à-dire qu'elles mobilisent des ressources dans une diversité de situations et amènent également aux compétences dans plusieurs disciplines. Il s'agit des compétences transférables " ailleurs » et " plus tard ». La transférabilité consiste à repérer dans de nouvelles situations des analogies avec des situations connues.

La notion de compétence transversale trouve de plus en plus sa place dans le domaine de l'éducation et la formation. Dans de nombreux pays, l'éducation nationale s'ouvre à cette notion qui voit, évidemment, son reflet dans la formation en langues.

Certes, il existe plusieurs typologies de compétences transversales et stratégiques basées sur divers critères: linguistiques, sociologiques, psychologiques, pédagogiques etc. Les actions d'Oxford des années 80 et 90 figurent parmi les contributions significatives à la recherche et la diffusion des connaissances en matière. Quoique ces travaux soient orientés avant tout à la description des stratégies utilisées par les apprenants lors de leur apprentissage des langues, le parallélisme de celles-ci aux compétences paraît évident. Par la suite, la nécessité de leur popularisation parmi les enseignants s'avère utile. La typologie de Rubin (Cyr, 1998 : 36) présentant un ensemble d'opérations pour amener l'apprenant à saisir ou comprendre la langue cible, contient :

- des stratégies de clarification et de vérification;

- des stratégies d'inférence;

- des stratégies de raisonnement déductif;

- des stratégies de ressourcement.

Bien que Rubin soit considéré comme le père des premières tentatives dans le domaine, nous permettons de juger cette répartition assez incomplète. Par contre, nous trouvons la classification de ses successeurs, celle de O'Malley et Chamot (Cyr, 1998 : 39) plus utilisable tant pour la recherche que pour les praticiens. Les deux auteurs, s'inspirant de la recherche en psychologie et en éducation, postulent que les stratégies d'apprentissage de la deuxième langue peuvent être groupées en trois catégories:

- stratégies métacognitives,

- stratégies cognitives, 
- stratégies socio-affectives.

Les premières stratégies impliquent une réflexion sur le processus d'apprentissage, y compris l'anticipation et la planification, l'autogestion et l'autorégulation, l'identification du problème et l'autoévaluation. Les stratégies cognitives représentent une manipulation mentale ou physique avec la matière à l'étude. On peut y comprendre l'application de techniques spécifiques pour exécuter la tâche, par exemple la répétition, la déduction, la traduction, l'inférence. L'interaction avec l'autrui, dans le but de favoriser l'apprentissage, appartient au champ socio-affectif. A part des stratégies coopératives, c'est le contrôle des émotions qui accompagne le procédé de l'apprentissage (idem : 36).

\subsection{Compétences transversales versus compétences disciplinaires}

Selon les auteurs de l'école québécoise (Langouche, 1996 : 24), les compétences transversales sont souvent mises en tension avec les savoirs disciplinaires. Pour ces chercheurs, l'acquisition de savoirs disciplinaires comprend la compréhension des explications, l'argumentation, la formulation des hypothèses, la recherche des solutions dans le cadre de la discipline donnée. En revanche, l'acquisition de compétences transversales est développée et appliquée globalement dans une diversité de disciplines. C'est pourquoi elle nécessite des tâches ouvertes et complexes ainsi qu'une attitude pédagogique adaptée. Des compétences transversales dépassent les frontières des savoirs disciplinaires tout en accentuant leur réinvestissement dans les situations concrètes de la vie.

En bref, il est impossible d'exercer une compétence transversale sans contenu, ainsi qu'on ne peut travailler un contenu sans exercer des compétences transversales. Il est plus efficace de fournir aux étudiants des connaissances préalables spécifiques avant de les entraîner à des démarches de transfert. Par exemple aux cours de langues appliquées, il vaut mieux que des notions de base en sciences économiques en langue maternelle devancent l'enseignement des matières économiques dispensées en langue étrangère.

\section{Compétences du point de vue didactique}

\subsection{Milieu académique en voie de changement}

Avant de traiter la problématique de la mise en place de l'approche par compétences dans le milieu universitaire, il nous semble important d'introduire quelques remarques à propos des changements de ce milieu au cours de dernières décennies. Aujourd'hui, comme dans le passé, l'université présente les caractéristiques d'une organisation structurée et mouvante. La structuration est représentée par les facultés, les unités de formation et de recherche, les départements, les orientations. La mouvance est due aux pressions des changements: scientifique, technologique, économique, politique ou idéologique. ${ }^{1}$ La création de l'espace européen de l'enseignement supérieur a apporté de nouveaux défis même pour les établissements supérieurs. Par suite, les objectifs prioritaires dans le domaine éducatif

${ }^{1}$ Les ouvrages de Touraine et Clozier sur le fonctionnement de la société et des organisations analysent le système universitaire de « différents champs qui s'interpénètrent et s'influencent mutuellement tout en gardant leur spécificité » (Dupont, 1994 : 94). Le champ sociologique qui est imprégné des valeurs privilégiées de l'époque, des relations humaines, de la représentation de la vie dans la période envisagée; le champ scientifique: inventions, découvertes, innovations, qui modifie ce champ sociologique; le champ politique qui met en forme le système universitaire; le champ pédagogique qui comprend l'enseignement universitaire avec des stratégies et des méthodologies appropriées (ibid.). 
et scientifique consistent à promouvoir l'internationalité, l'interdisciplinarité et la compétitivité. La nécessité de dépasser les limites étroites des disciplines académiques a apporté la question de la transdisciplinarité. Celle-ci devrait se refléter dans la prise d'information à de multiples niveaux, dans la perception d'un problème sous des angles interdisciplinaires, dans la jonction même du savoir scientifique et du savoir social (Dupont, 1994 : 100).

Ces changements ont naturellement pour conséquence une pédagogie innovatrice ainsi qu'une flexibilité universitaire qui suscite une véritable liaison entre la théorie et la pratique. Il en ressort que la formation universitaire vise le double objectif: celui d'intégrer les étudiants dans le monde universitaire du pays respectif, éventuellement, de préparer des séjours d'études à l'étranger, et parallèlement, celui de professionnaliser des contenus curriculaires pour garantir une meilleure insertion des diplômés sur le marché du travail.

Le rôle du pédagogue de langue à l'université est également altéré. Les enseignants deviennent plus responsables des programmes d'études élaborés en conformité avec des programmes similaires chez leurs partenaires. L'harmonisation des programmes communs à plusieurs universités, y compris à l'étranger, et la coopération interuniversitaire à divers niveaux s'affirment prioritaires. D'autant plus, la nécessité de promouvoir des mobilités d'enseignants, des rencontres avec des collègues des universités ou institutions partenaires lors des colloques et conférences scientifiques, mais aussi des entretiens au niveau bilatéral ou multilatéral entre des collaborateurs potentiels des projets internationaux communs devient incontournable.

D'ailleurs, l'orientation sur l'apprenant préparant sa carrière professionnelle, oblige à réfléchir sur nombreuses questions: Quelles compétences développer davantage et comment les faire apprendre aux étudiants? Comment mettre en accord la matière avec la compétence ciblée ? Quelles méthodes didactiques exploiter pour rendre les étudiants plus autonomes et plus compétents ? etc.

Il est à noter que dans la situation d'appliquer une nouvelle approche pédagogique, on prévoit également de former un nouvel étudiant. Cela signifie sa transformation de l'auditeur passif à l'acteur de son propre apprentissage qui prendra des initiatives, partagera ses connaissances et ses expériences avec les autres et appliquera des stratégies d'apprentissage qui lui seront les plus efficaces.

Actuellement, les pédagogues exploitent de nombreuses méthodes d'enseignement moderne, soit d'une manière ciblée, soit inconsciente. Comme c'était mentionné ci-dessus, le processus pédagogique devrait mobiliser chez l'apprenant des savoirs et des savoir-faire, et cela en rapport réciproque. Quoique l'enseignant ait la tendance de cibler une compétence concrète, il devrait toutefois prévoir la corrélation de celle-ci avec une autre. La compétence repose sur la mobilisation des ressources propres à l'individu et également de celles qui proviennent de son environnement. Le processus de formation supérieure devrait mobiliser et développer ces deux types de ressources, et cela dans une diversité de situations, et à travers un processus d'adaptation.

2.2 Compétences transversales à développer lors des cours de langues étrangères appliquées (LEA)

Dans les conditions de la formation universitaire slovaque, il existe actuellement une filière des LEA (à la Faculté des langues appliquées) qui a son siège à l'Université d'Economie à Bratislava. Depuis sa création en 2010, elle forme de futurs cadres bilingues, spécialisés en sciences économiques et en communication interculturelle. Il s'agit donc d'une formation pluridisciplinaire recouvrant des disciplines linguistiques et des matières d'application. En ce qui concerne la formation en spécialité anglais - français, les enseignements portent sur les deux langues et civilisations étrangères, et sur les matières d'application (microéconomie, 
macroéconomie, management, marketing, économie mondiale et la politologie). Les étudiants optent pour une troisième langue étrangère pour en acquérir les notions de base.

Comme l'enseignement des langues fait partie intégrante du processus d'internationalisation, il faut qu'il cède la place à l'organisation d'un environnement d'apprentissage permettant aux étudiants de développer leurs compétences pour communiquer couramment avec des natifs, parler et débattre en public, savoir nouer et développer des contacts personnels et professionnels, comprendre les différences culturelles et savoir s'y adapter. Tatiana Hrivíková, enseignante en LEA, déclare que pour des étudiants slovaques «c'est la capacité de réussir ses études dans un environnement de langue et culture étrangères qui devient souvent un facteur limitant. Bien que les étudiants soient intelligents et responsables, ils n'osent pas demander une bourse à l'étranger, rien que par des craintes de manque de compétences langagières... En outre, des enseignants qui dispensent des matières en langues étrangères, eux aussi, ressentent un besoin impératif de regagner la confiance en leur maîtrise de langue. Afin de pouvoir mener des cours magistraux et des travaux dirigés avec un certain degré de spontanéité et $d$ 'aisance, ils auraient forcément besoin des stages linguistiques, professionnels et pédagogiques » (trad. aut.) (Hriviková, 2013 :104). Nous ne sommes pas étonnés que les pédagogues ne voient pas leur perspective trop optimiste, vu des restrictions des ressources financières pour les universités.

Dans l'enseignement transdisciplinaire, des thèmes sont traités de point de vue de plusieurs disciplines. Tel procédé exige une étroite collaboration de l'équipe enseignante ainsi qu'une bonne coordination des contenus et des méthodes de travail. L'organisation d'un cours doit reposer sur un enseignement actif. Par conséquent, les activités de la classe s'organisent en fonction de la tâche à accomplir, de sorte que les actes de parole, la grammaire, le lexique, la prononciation et l'orthographe deviennent des moyens langagiers nécessaires au développement des compétences communicatives et, si possible, transversales. L'intégration des éléments linguistiques dans les activités de communication et le transfert d'application de connaissances et de capacités dans de nouveaux contextes sont des atouts majeurs de la formation en LEA. Dans cette perspective, il est utile de combiner un apprentissage cognitif et un apprentissage actif proche du vécu des jeunes étudiants. Mais en même temps, c'est une tâche ardue pour l'enseignant qui ne fait plus apprendre à ses étudiants des notions, des concepts et des théories. Par contre, il leur offre des conseils et fixe des objectifs graduels pour encourager le développement de leurs compétences. Le pédagogue devient un conseiller, un coach, car il ne transmet pas de savoirs, mais il amène à les construire et les appliquer de façon adéquate. Dans cette approche, une bonne ambiance représente une des conditions de la réussite et de la satisfaction sur les deux côtés.

En nous appuyant sur la classification des stratégies d'apprentissage formulée par O’Malley et Chamot (Cyr, 1998 : 39), nous apportons une vue synoptique sur des compétences et des stratégies que nous trouvons pertinentes. Quoiqu'il ne soit pas toujours facile à établir la frontière exacte parmi les types de compétences, nous avons décidé pourtant de les répartir en quatre grandes catégories:

- des compétences cognitives,

- des compétences métacognitives,

- des compétences socio-affectives

- des compétences informatiques. 
Le tableau 1 présente des compétences transversales susceptibles d'être abordées et développées lors des cours de langues et exploitées dans les études universitaires. ${ }^{1}$ Loin de fournir la liste exhaustive de compétences, nous visons particulièrement celles qui, en lien avec des stratégies d'apprentissage, peuvent mener les apprentis à une étude plus autonome et plus efficace.

Tableau 1. Compétences et stratégies à développer lors des cours de langues dans le milieu universitaire.

\begin{tabular}{|c|c|c|}
\hline Catégorie & Compétence & Stratégies pour développer la compétence \\
\hline \multirow[t]{3}{*}{$\begin{array}{l}\text { Compétences } \\
\text { cognitives }\end{array}$} & $\begin{array}{l}\text { Saisir et } \\
\text { comprendre } \\
\text { l'information }\end{array}$ & $\begin{array}{ll}> & \text { s'entraîner à la lecture active } \\
& \text { prendre des notes } \\
& \text { dégager le thème et la problématique } \\
> & \text { percevoir l'idée principale } \\
> & \text { déchiffrer le texte et le paratexte } \\
> & \text { dégager le contexte et l'intention } \\
> & \text { comprendre l'acte d'énonciation } \\
> & \text { identifier l'information explicite et } \\
& \text { implicite } \\
> & \text { faire des associations, des analogies } \\
> & \text { déterminer le type et le genre du texte } \\
> & \text { saisir le plan du discours } \\
> & \text { comprendre et reformuler la consigne, le } \\
& \text { message, l'explication, l'argumentation etc. } \\
> & \text { établir le champ sémantique }\end{array}$ \\
\hline & $\begin{array}{l}\text { Exploiter } \\
\text { l'information }\end{array}$ & $\begin{array}{l}>\text { utiliser des stratégies de recherche efficace } \\
>\text { évaluer des sources d'information } \\
>\text { analyser des informations de façon critique } \\
>\text { organiser des informations de façon } \\
\text { cohérente } \\
>\text { formuler la thèse, l'antithèse et la synthèse } \\
>\text { utiliser l'information dans divers contextes } \\
>\text { reproduire, reformuler, résumer des propos } \\
>\text { citer et paraphraser des idées de l'autrui } \\
>\text { construire une fiche de lecture } \\
>\text { formuler la problématique au sein d'un } \\
>\text { sujet } \\
>\text { traduire des expressions, des phrases } \\
>\text { produire une synthèse de documents } \\
>\text { rédiger un exposé cohérent } \\
>\text { répondre à un questionnaire }\end{array}$ \\
\hline & $\begin{array}{l}\text { Résoudre un } \\
\text { problème }\end{array}$ & $\begin{array}{l}>\text { analyser des données } \\
>\text { ressortir les divers aspects du problème } \\
>\text { définir les tâches pour résoudre le } \\
\text { problème } \\
>\text { identifier l'objet de la tâche } \\
>\quad \text { planifier des tâches }\end{array}$ \\
\hline
\end{tabular}

${ }^{1}$ L'argumentation est ressortie des expériences avec le système d'enseignement universitaire slovaque. 


\begin{tabular}{|c|c|c|}
\hline & & $\begin{array}{l}>\text { comparer des attitudes } \\
>\quad \text { prendre des décisions } \\
>\quad \text { résumer, présenter des résultats }\end{array}$ \\
\hline & $\begin{array}{l}\text { Manifester son } \\
\text { esprit créatif }\end{array}$ & $\begin{array}{l}>\text { trouver de nouvelles idées et/ou approches } \\
>\text { créer des propositions } \\
>\text { explorer de nouvelles façons de faire } \\
>\text { démontrer la tolérance } \\
>\text { établir des relations entre les éléments } \\
>\text { trouver des arguments et contre-arguments } \\
>\text { élabonner, déduire } \\
>\text { illustrer des projets } \\
>\text { introduire et conclure la réflexion }\end{array}$ \\
\hline & $\begin{array}{l}\text { Manifester son } \\
\text { jugement critique }\end{array}$ & $\begin{array}{l}>\text { formuler des questions et y répondre } \\
>\text { utiliser des critères d'appréciation } \\
>\text { développer son point de vue } \\
>\text { nuancer son jugement } \\
>\text { être ouvert à la remise en question }\end{array}$ \\
\hline & $\begin{array}{l}\text { Communiquer de } \\
\text { manière } \\
\text { appropriée }\end{array}$ & $\begin{array}{ll}> & \text { employer le vocabulaire conforme à la } \\
& \text { norme } \\
> & \text { respecter des règles de grammaire } \\
> & \text { respecter les usages et les conventions } \\
> & \text { adapter son langage à une situation donnée } \\
& \text { (le contexte et l'interlocuteur) } \\
> & \text { communiquer d'une façon cohérente } \\
> & \text { utiliser des connecteurs logiques }\end{array}$ \\
\hline $\begin{array}{l}\text { Compétences } \\
\text { métacognitive } \\
\mathrm{s}\end{array}$ & 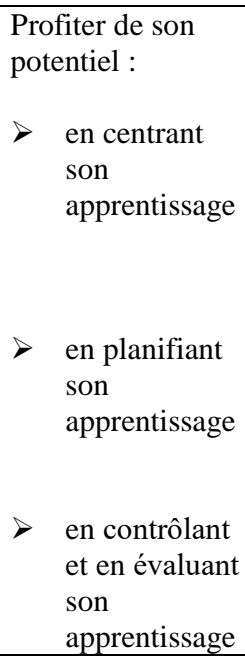 & $\begin{array}{l}>\text { examiner la matière à apprendre } \\
>\text { se rendre compte des liens avec le connu } \\
>\text { se concentrer sur la compréhension } \\
>\text { se fixer des buts } \\
>\text { planifier l'exécution des tâches } \\
>\text { identifier ses possibilités et ses limites } \\
>\text { apprécier l'impact de ses actions } \\
>\text { être autonome dans l'expression } \\
>\text { poursuivre des buts fixés } \\
>\text { se déterminer des critères d'évaluation } \\
>\text { s'autocontrôler } \\
>\text { s'autocorriger } \\
>\text { s'autoévaluer }\end{array}$ \\
\hline $\begin{array}{l}\text { Compétences } \\
\text { sociales }\end{array}$ & $\begin{array}{l}\text { Coopérer } \\
\text { Cultiver }\end{array}$ & $\begin{array}{ll}> & \text { s'engager dans un travail de groupe } \\
> & \text { respecter des règles communes } \\
> & \text { reconnaître les caractéristiques des autres } \\
> & \text { prendre en compte le point de vue adverse } \\
> & \text { solliciter des clarifications }\end{array}$ \\
\hline
\end{tabular}




\begin{tabular}{|c|c|c|}
\hline & l'empathie & $\begin{array}{ll}> & \text { gérer des conflits } \\
> & \text { partager des perceptions et des valeurs } \\
> & \text { s'ouvrir aux autres cultures }\end{array}$ \\
\hline $\begin{array}{l}\text { Compétences } \\
\text { affectives }\end{array}$ & $\begin{array}{l}\text { S'auto-encourager } \\
\text { S'autoévaluer } \\
\text { S'autocontrôler }\end{array}$ & $\begin{array}{l}>\text { vaincre l'anxiété } \\
>\text { se féliciter de la réussite } \\
>\text { contrôler ses émotions } \\
>\text { partager ses sentiments }\end{array}$ \\
\hline $\begin{array}{l}\text { Compétences } \\
\text { informatiques }\end{array}$ & $\begin{array}{l}\text { Maîtriser et } \\
\text { exploiter } \\
\text { les nouvelles } \\
\text { technologies } \\
\text { d'information et } \\
\text { de communication }\end{array}$ & $\begin{array}{ll}> & \text { utiliser des ressources technologiques } \\
& \text { variées } \\
> & \text { respecter des règles d'éthique }\end{array}$ \\
\hline
\end{tabular}

Dans le milieu universitaire, le développement de compétences transversales à dominante cognitive touche avant tout le domaine de connaissances déclaratives, ainsi que la capacité de saisir, comprendre et traiter l'information. On y voit un trait interdisciplinaire, étant donné que chaque matière ou discipline nécessite le traitement des informations de la part de l'étudiant, peu importe si elles sont transmises à l'oral ou à l'écrit. L'étudiant développe toutes les démarches mises en œuvre pour prendre des notes, retirer des idées principales d'un texte, mémoriser et réutiliser des connaissances dans de nouveaux contextes etc.

Dans le cadre des langues appliquées les étudiants sont menés à exercer des activités conduisant à la compréhension et à la production de textes ou discours de spécialité. Leurs études aboutissent, à part des épreuves orales, à la rédaction du mémoire de licence (en fin du sixième semestre) et mémoire de maîtrise (en fin du dixième semestre). Les thèmes de mémoires varient en fonction de spécialité, préférences et options du candidat. C'est la situation où la transdisciplinarité s'affirme complètement. Les enseignants prennent le rôle de tuteurs afin de conseiller, aider les jeunes à sélectionner un thème, à exploiter des stratégies de recherche convenables, à traiter des informations d'une manière appropriée. Lors d'épreuves finales, les étudiants ont à manifester leurs compétences d'analyser, synthétiser, argumenter, justifier, présenter l'esprit critique tout en gardant une certaine objectivité.

Une des matières enseignées au sein du programme d'études de langues étrangères appliquées a pour objectif d'aborder le discours universitaire. ${ }^{1}$ Les cours visent à développer progressivement la compréhension des textes de spécialité (sciences sociales et économiques), à rédiger un résumé ou un compte rendu, pour préparer des étudiants, au fur et à mesure, à la production orale et écrite des discours aux sujets de spécialité. On procède de la compréhension globale d'un texte ou d'un acte de communication à la compréhension plus détaillée, et cela à l'aide de l'inférence. La capacité d'inférer le sens de nouveaux éléments dépend de chaque individu. On peut l'observer par exemple à travers la prise de notes, le repérage des mots-clés et des idées essentielles ou bien l'établissement des champs lexicaux. L'apprenant est amené à regrouper et classer des éléments selon diverses catégories, à trouver des analogies. Celui qui a besoin de consulter constamment le dictionnaire ou

${ }^{1}$ Dans le présent article, nous avons recours à l'expérience acquise lors des cours de Français sur objectifs universitaires du programme d'études «Langues étrangères et la civilisation interculturelle » dispensé à la Faculté des langues appliquées à l'Université d'Economie à Bratislava, depuis 2010. 
vérifier le vocabulaire ne fait pas d'inférence. Il est probable que l'étudiant n'ayant pas de capacité d'inférer ne l'ait pas appris lors de ses études précédentes.

Avant d'accomplir la tâche plus complexe - rédiger le mémoire et le soutenir, les étudiants s'exercent en préparant la dissertation, la synthèse de documents, le projet de mémoire, présentent des exposés et s'entraînent à la prise de parole en public. La plupart de ces activités sont réalisées à travers des disciplines enseignées suivantes (jusqu'ici au niveau de licence) : la langue sur objectifs spécifiques, la langue sur objectifs universitaires, la communication interculturelle, les négociations commerciales, l'introduction à la traduction et l'interprétariat et la stylistique appliquée. Une préparation interdisciplinaire, au niveau des contenus, de stratégies et des méthodes de travail est donc inévitable.

Sur le plan des méthodes, la déduction est assez fréquente lorsqu'il s'agit d'appliquer une règle en vue de compréhension ou de production d'un discours. Bien qu'elle puisse mener parfois à une généralisation automatique, c'est une opération qui semble très productive car elle démontre que l'apprenant est capable de générer des règles, par exemple celles du fonctionnement de la langue.

La recherche documentaire appartient parmi les stratégies fréquemment exercées notamment dans ce milieu. Elle consiste à exploiter des sources de références, à chercher la signification de nouvelles expressions, parfois à faire la traduction. La stratégie de substituer et paraphraser le vocabulaire, les syntagmes, les phrases inconnus par d'autres mots ou formulations en langue cible, est un moyen pour vérifier des savoirs lexicales et grammaticales, de même que la compétence d'associer ou d'inférer la signification d'une unité linguistique ou énonciative. En effet, «en traduisant il faut prêter l'attention au choix de tels équivalents qui correspondent au texte en langue initiale, et qui en même temps, reflètent la réalité dénommée de telle façon qu'elle soit appréhendée également par des lecteurs de la différente communauté culturelle» (trad. aut.) (Melušová, 2014 : 281). Certains didacticiens constatent que «l'utilisation directe d'un mot de la première langue en vue de produire un énoncé dans la deuxième langue n'est considérée comme une stratégie d'apprentissage mais plutôt comme une stratégie de communication de type compensatoire » (Cyr, 1998: 53). Il est vrai que l'apprenant se sent plus rassuré de consulter des dictionnaires bilingues, mais pourtant, il vaut mieux qu'il s'adapte progressivement à consulter le dictionnaire unilingue en langue cible.

En résumant, nous partageons l'idée que dans les langues comme „dans le cadre des sciences humaines, il existe actuellement une tendance d'appliquer une approche complexe, holistique et universelle. Pour ne pas tomber dans l'inefficacité ou l'isolement, la problématique du concept interdisciplinaire de la matière enseignée est incontournable » (trad. aut.) (Dulebová, 2013:13).

Au niveau de la coopération au sein du groupe des apprentis, la réalisation de la pédagogie $d u$ projet nous semble particulièrement efficace à développer des compétences transversales. Il s'agit de l'approche qui accorde une place significative au travail coopératif. Les individus réunis par une tâche commune à accomplir, c'està-dire l'élaboration d'un projet, sont obligés d'échanger: lors de remue-méninges, lorsqu'ils formulent et analysent un problème ou expriment leurs opinions. En plus, ils gèrent des conflits pour trouver un consensus, planifient et organisent ensemble des tâches ou discutent des méthodes de travail etc.

À titre d'exemple, nous pouvons nommer le projet «Mini-colloque » réalisé en deuxième année de licence au sein de la faculté, ${ }^{1}$ qui suscite à chaque fois l'intérêt auprès des groupes d'étudiants en LEA. Le projet consiste en quatre phases

${ }^{1}$ Faculté des langues appliquées à l’Université d'Economie à Bratislava 
principales: celle préparation, d'organisation, de réalisation (simulation) et d'évaluation. Toutes les activités à faire font appel aux méthodes de coopération, et cela dans des situations où les apprenants déterminent des buts à atteindre, organisent le travail collectif, cherchent des solutions, échangent des attitudes. En outre, ils apprennent à adapter leur manière de s'exprimer, à utiliser différents registres de langue pour parler aux différents interlocuteurs (compagnons de classe - professeurs, individu - public). Des compétences transversales sont sollicitées également en situation d'interprétation et d'évaluation des résultats du projet.

A première vue, il peut paraître que le rôle d'enseignant soit inhibé et que toute action se déroule spontanément. Bien que nous ne puissions pas nier la présence d'improvisation tant de la part des apprentis que de la part de l'enseignant durant le cours, il est certain que la réussite pédagogique dépend de la planification des situations d'apprentissage susceptibles de développer des compétences transversales. Pour que l'apprenant soit capable de se référer à celles-ci, il vaut mieux le préparer systématiquement à travers plusieurs disciplines et tout au long de ses études.

Le développement social et affectif représente des comportements et des attitudes favorables envers l'autrui. L'encouragement du savoir-être favorise la structuration de l'identité de l'individu, et en même temps, son ouverture aux autres cultures. La communication en langues étrangères est un moyen favorable à exprimer ses opinions et attitudes à l'égard des thèmes de société actuelle. Il est donc conseillé d'introduire également la problématique d'identités et d'en ouvrir les débats au cours de langues. L'estime de soi et de l'autrui sont des sujets à analyser avec des jeunes d'autant plus que ces phénomènes identitaires ayant un rapport avec globalisation, multiculturalisme, immigration et conflits internationaux résonnent dans notre vie quotidienne. «En Slovaquie, il existe des universités qui respectent en entier les principes de l'interculturel et multiculturel, basés sur le dialogue des cultures, dans leurs programmes orientés en langues étrangères » (trad. aut.) (Kvapil, 2014 : 7). Aujourd'hui plus qu'auparavant, l'université devrait développer la compétence interculturelle chez ses étudiants - futurs entrepreneurs, employés ou manageurs, diplomates. Il appartient à l'école d'encourager les jeunes à développer leur capacité d'apprendre à l'aide des expériences d'autres langues et d'autres cultures (trad. aut.) (Birova - Eliasova, 2014 : 76).

2.3 A propos des contraintes rencontrées lors de la mise en œuvre de la pédagogie par compétences

Il nous semble qu'aujourd'hui, les étudiants qui entrent à l'université pratiquent peu les langues étrangères. Au cours de la première année d'études supérieures, ils éprouvent encore des difficultés à réaliser la lecture active, à prendre des notes, à pénétrer le sens d'un texte. Ils préfèrent la mémorisation à l'analyse critique. Il n'est pas rare qu'ils arrivent à l'université sans avoir le projet précis, sans savoir utiliser des formes d'apprentissage individuel. Nos étudiants slovaques rencontrent certaines difficultés liées aux activités réceptives, comme par exemple à prendre des notes à partir du discours écouté, à dégager l'idée directrice du texte, à comprendre l'essentiel et l'interpréter. Ils ressentent des problèmes s'ils doivent s'orienter dans un enchevêtrement d'informations à trier et à organiser d'une façon logique. Quelquefois, ils n'arrivent pas à trouver des arguments pertinants pour justifier leur opinion ou persuader leur interlocuteur. En outre, certaines activités de production, comme reformuler, exposer des idées, argumenter, articuler logiquement le discours, peuvent soulever un problème. Tout cela inclut la compétence à porter un jugement critique dans le processus de formation, par exemple dans le cadre de la prise de décisions, dans le travail d'équipe, dans la façon de considérer les différentes perspectives. Mais de l'autre côté, il faut avouer que la jeune génération ne manque ni d'invention ni de

41

XLinguae Journal, Volume 8 Issue 4, October 2015, ISSN 1337-8384 
créativité. Les jeunes savent nous surprendre d'une façon positive lorsqu'ils ont à traiter un sujet qu'ils trouvent proche et intéressant ou encore applicable dans leur vie professionnelle. En bref, pour ce qui est à développer davantage auprès des étudiants à l'université slovaque, ce sont des stratégies de recherche et de traitement de données, la réflexion et l'analyse critique et l'apprentissage dynamique.

Nous observons encore des traces de la pédagogie traditionnelle qui avait formé le type d'étudiant plutôt passif, tant dans ses apprentissages que dans sa motivation et autorégulation. Jusqu'ici, c'était à l'enseignant de sensibiliser son étudiant à réfléchir sur la rentabilité des stratégies selon les tâches proposées. À présent, nous faisons des efforts pour que nos étudiants deviennent capables de porter un regard critique sur leur travail et leurs stratégies d'apprentissage. Il n'est jamais trop tard pour apprendre à identifier des moyens pour améliorer leur performance ou, le cas échéant, comprendre la raison de leur échec.

Il faut avouer que les causes de défaillance de la pédagogie par compétences sont multiples. Si l'université privilégie la transmission linéaire des connaissances, à savoir : d'abord la présentation, ensuite la réception, les apprentis prennent le rôle des spectateurs ou auditeurs passifs. Au surplus, le cloisonnement des matières enseignées peut causer l'absence d'un vécu intellectuel qui présente un des éléments importants pour satisfaire les attentes des étudiants universitaires.

Le progrès en pédagogie des langues apporte naturellement le changement au champ de l'évaluation. Selon Yiru Xu «le structuralisme, qui insiste sur la séparation des éléments linguistiques dans l'évaluation afin d'assurer l'objectivité, la fiabilité et la validité, évolue petit à petit vers l'approche communicative » (Xu, 2014: 4). Sur le plan d'évaluation, plusieurs erreurs peuvent surgir, étant donné que la pratique d'évaluer en situation, c'est-à-dire au fil des activités réalisées en classe, est assez peu répandue. Evaluer des compétences transversales est plus difficile qu'exercer une évaluation des savoirs. La pratique de telle évaluation formative exige le changement de toute la situation d'apprentissage, y compris des objectifs, des activités, des critères. Il est indispensable de donner la priorité à l'évaluation du processus d'apprentissage plutôt que de ses résultats.

Il est évident que dans l'apprentissage, il n'existe pas une seule bonne stratégie. La stratégie satisfaisant un apprenti, ne doit pas convenir à un autre, comme elle dépend des caractéristiques cognitives et affectives de chaque individu. En outre, les stratégies varient en fonction du contexte, du contenu, de la motivation, de l'âge etc. C'est pourquoi un bon enseignant tient à proposer à ses étudiants des occasions de réfléchir sur leurs propres stratégies pour en repérer les points forts et les défauts.

Malgré ces obstacles mentionnés qui freinent le renouveau pédagogique, nous sommes persuadés que dans le cadre de l'enseignement universitaire, il est incontournable de favoriser la mise en place de la manière (des compétences transversales) qui compte plus que la matière (la discipline).

\section{Conclusion}

Finalement, on peut constater que les compétences transversales constituent l'un des aspects dominants des changements dans les pratiques éducatives parce qu'elles conduisent plutôt à découvrir, à créer et à développer, ce qui s'avère primordiale pour pouvoir articuler la théorie avec la pratique. C'est pourquoi l'enseignement universitaire, lui aussi, devrait inciter à développer des compétences qui s'exercent dans des contextes diversifiés. L'autonomie, la créativité et les compétences transversales ne peuvent pas être l'affaire d'une discipline.

Mais de l'autre côté, implanter la formation par compétences n'est pas du tout aisé, puisqu'il faut repenser l'ensemble du système, des programmes, des supports, des outils d'enseignement de même que des procédures d'évaluation. Plusieurs 
exemples démontrent l'ampleur de la réforme pédagogique introduite dans la plupart des pays européens. On y voit qu'elle n'est pas possible sans innover notamment les attitudes des acteurs du processus pédagogique, c'est-à-dire des enseignants et des étudiants. Pour motiver davantage les pédagogues au travail tellement exigeant, les compétents nationaux et européens devraient œuvrer pour rendre la profession d'enseignant plus attractive. Néanmoins, la responsabilité d'enseigner les compétences transversales doit être celle de l'école dans sa globalité et non seulement celle de quelques enseignants passionnés, étant donné que les compétences doivent être acquises lors de la formation initiale, et développées tout au long de la vie.

En Slovaquie plusieurs documents officiels insistent sur l'importance d'introduire dans la pratique pédagogique des stratégies basées sur les compétences et, par la suite, un bon nombre de monographies et articles à ce sujet ont été publiés. Malgré ces efforts des théoriciens, didacticiens et formateurs, nous nous rendons compte que telle réforme importante devrait concerner davantage ceux qui sont en rapport direct avec les jeunes - les enseignants.

À part des tentatives de quelques enseignants à évoluer leurs pratiques didactiques dans ce sens, à l'heure actuelle, on ne peut pas parler de la mise en œuvre de la pédagogie par compétences dans le système d'enseignement supérieur slovaque. Un référentiel de compétences et des efforts plutôt isolés ne sont pas suffisants. La révision globale des curricula, une élucidation des compétences à évaluer et des procédures d'évaluation, ainsi qu'une formation approfondie des enseignants pourront conduire à atteindre l'objectif tellement exigeant. Quant à l'enseignement des langues au niveau universitaire, il faudrait se focaliser sur les objectifs bien définis, la mise en cohérence de diverses disciplines et les formes d'évaluation plus efficaces. Tout cela suppose une réflexion commune de toutes les parties engagées.

Ces changements à plusieurs niveaux tiendront à ce que les compétences soient développées et évaluées à travers des situations concrètes et proches de la vie réelle. Si l'objectif de renouveler les démarches pédagogiques, les outils, les stratégies est déjà déterminé, la mise en œuvre complexe de l'approche par compétences devra évoluer dans les années à venir.

\section{Bibliographic references}

AFRIAT, CH. - GAY, C. - LOISIL, F. 2006. Mobilites professionnelles et competences transversales [cit. 03.03.2015]. Available online

< www.ladocumentationfrancaise.fr/var/storage/rapports-publics/06400052 7.pdf>

Approche des compétences transversales en Communauté française et au SIREAS/FAE dans le cadre de la formation professionnelle modulaire et en alternance [cit. 03.03.2015]. Available online <http://www.cdoc-alpha.be>

BEKOVA, L.- GRAJCAR, S. (2012) Zrucnosti pre riadenie vlastnej vzdelavacej a profesijnej drahy a ich rozvoj v sektore vzdelavania SAAIC, Slovenska akademicka asociacia pre medzinárodnu spolupracu, centrum Euroguidance. Bratislava 2012. ISBN 978-80-89517-06-0.

BIROVA, J. - ELIASOVA S. 2014. Plurilingual and Pluricultural Competence and Foreign Language Teaching at Primary and Secondary Schools in Slovakia [Viacjazyčna a plurikulturna kompetencia a vyucovanie cudzich jazykov na zakladnych a strednych skolach v Slovenskej republike]. In: XLinguae - European Scientific Language Journal. ISSN 1337-8384, Vol. 7, no. 1, pp. 75-82.

CESAR, A. - PIRARD, F. 2012. GLOSSAIRE Essai de clarification conceptuelle des notions utilisées dans le champ de l'accueil de l'enfance et des formations qui y sont liées. Liège: Recherche-action 114 [cit. 03.03.2015]. Available online <http://orbi.ulg.ac.be>

CHEVALIER, B. 1992. Lecture et prise de notes. Paris: Nathan. ISBN 209 190562-3. 
CYR, P. 1998. Les strategies d'apprentissage. Paris : CLE International. ISBN 209033 326-X.

De la transmission des savoirs à l'approche par competences. Dossier d'actualite (ancien titre : Lettre d'information) $\mathrm{n}^{\circ} 34$ - avril 2008 [cit. 03 .03.2015]. Available online <http://ife.ens-lyon.fr/vst/DA-Veille/34-avril-2008.pdf>

DULEBOVA, I. 2013. Interdisciplinarny prístup vo vyučovani lingvorealii ruskeho arealu. In Forum cudzich jazykov, roč. 5, č. 2, pp. 7-14. ISSN 1337-9321.

HOFFLACK, J. Vzdelavanie pomocou kompetencii a skolska reforma v Belgicku, vo francuzskej komunite [cit. 03.03.2015]. Available online <www2.statpedu.sk/buxus/docs/sys_vzdel_v_zahranici/system_vzdelavania_Belgic ko.pdf>

HOUCHOT, A. - ROBIN, F. 2007. Les livrets de competences: Nouveaux outils pour l'evaluation des acquis. $\mathrm{N}^{\circ}$ 2007-048. Rapport a monsieur le ministre de l'Education nationale.

HRIVIKOVA, T. 2013. Vedecky vyskum a vyuka jazyku v komunikacni sebereflexe a kompetence. Scientific Research and the Teaching of Foreign Languages V. Hradec Kralove, 2013. ISBN 978-80-7435-323-9. pp. 100-105.

PERY-WOODLEY, M.- P. 1993. Les ecrits dans l'apprentissage. Paris : HachetteLivre. ISBN 2-01-020549-9.

DUPONT, P. - OSSANDON, M. 1994. La pedagogie universitaire. Paris : Presses universitaires de France. ISBN 2130465455.

KVAPIL, R. 2014. Od realii k interkulturnej kompetencii ucitela - rusistu. Bratislava: MPC.

LANGOUCHE, A. S., PETIT V. - PHILIPPE, M. C. - ROMAINVILLE, M. 1996. Les competences transversales: Une incitation a faire apprendre a apprendre. Informations Pedagogiques $\mathrm{n}^{\circ} 24$, mars 1996 [cit. 03 .03.2015]. Available online <http://www.restode.cfwb.be/download/infoped/info24c.pdf> p. 24.

LANGEVIN, S. 2015. Les nouvelles technologies n'impliquent pas des innovations didactiques. In Le français dans le monde, numero 397, pp. 22-23. ISSN 0015-9395.

LAFORTUNE, L. 2010 Pedagogie du projet et developpement des competences transversales: un changement de posture pedagogique. Canadian education association [cit. 03 .03.2015]. Available online

<www.cea-ace.ca/sites/default/files/EdCan-2009-v49-n5-Lafortune.pdf>

LEGENDRE, F. Centre universitaire de recherche en sciences de l'éducation de Picardie, CURSEP-Université d'Amiens (en 2006). [cit. 03 .03.2015]. Available online <http://ife.ens-lyon.fr/vst/DA-Veille/34-avril-2008.pdf>

MELUSOVA, E. 2014. Specifika prekladu z francuzstiny do slovenciny. In Cudzie jazyky v premenach casu IV, Bratislava: Ekonom. ISBN 978-80-225-3905-0.

MOIRAND, S. 1979. Situation d'ecrit. Paris: Cle International. ISBN 2-19-033253-2.

Position sur la Communication de la Commission europeenne « Repenser l'education

- Investir dans les competences pour de meilleurs resultats socioeconomiques » Bruxelles, [cit. 03 .03.2015]. Available online 〈http://www.etuc.org/fr >.

POVCHANIC, S. 2009. Culture et communication interculturelle. Bratislava: Ekonom. ISBN 978-80-225-2684-5.

PROCIKOVA, J. - CAJKOVICOVA, J. (2014) Sprievodca studiom na Fakulte aplikovanych jazykov Ekonomickej university v Bratislave. Bratislava: Ekonóm. ISBN 978-80-225-3912-8.

Referentiel de competences pour le $2^{\mathrm{e}}$ et le $3^{\mathrm{e}}$ degres de l'enseignement general et technique de transition de l'enseignement secondaire ordinaire. Français premiere langue etrangere [cit. 03 .03.2015]. Available online

<http://www.bildungsserver.be>. 
RIZEKOVA, I. 2014. Le Francais sur objectifs universitaires. Bratislava: Ekonom. ISBN 978-80-225-3847-3.

RIZEKOVA, I. 2014. Vyucba argumentacnych postupov na hodinach odborneho cudzieho jazyka $\mathrm{v}$ akademickom prostredi. In Foreign language competence as an integral component of the university graduade profile. BRNO: Radek Nedoma. ISBN 978-80-7231-982-4, pp.274-28.

Socles de Competences, Enseignement fondamental et premier degre de l'enseignement secondaire, Ministere de la Communaute francaise, Bruxelles, 1999. Approche des competences transversales en Communaute francaise et au SIREAS/FAE dans le cadre de la formation professionnelle modulaire et en alternance [cit. 03.03 .2015$]$. Available online <http://www.cdoc-alpha.be>

Sprava o implementacii pracovneho programu Vzdelavanie a odborna priprava 2010. Ministerstvo skolstva [cit. 03 .03.2015]. Available online <https://www.minedu.sk/data/att/2326.pdf >.

Synthese de la legislation de l'UE [cit. 03 .03.2015]. Available online $<$ http://europa.eu/legislation_summaries/education_training_youth/lifelong_learning /c11088 fr.htm>

TREMBLAY, S. (2007) Soutenir l'appropriation des competences transversales et des domaines generaux de formation. Quebec: Bibliotheque et Archives nationales du Quebec. ISBN 978-2-550-49286-3. [cit. 03 .03.2015]. Available online <https://www.cse.gouv.qc.ca>.

XU, Y. 2014. Les competences communicatives dans l'evaluation de l'oral: le cas des debutants en francais langue etrangere. In: XLinguae Journal, Volume 7 Issue 3, June 2014, ISSN 1337-8384.

YAICHE, F. 1995. Grilles\&methodes de recherche d'idees. Sevres: BELC. ISBN 285420-257-0.

Competences transversales. Programme de formation de l'ecole quebecoise [cit. $03.03 .2015]$.

Available online

<www1.mels.gouv.qc.ca/sections/programmeFormation/secondaire2/medias/3pfeq_chap3.pdf>

Words: 7032

Characters: 50080 (27,82 standard pages)

PhDr. Iveta Rizeková, PhD.

Department of Romance and Slavic languages

Faculty of Applied Languages

University of Economics in Bratislava

Dolnozemská cesta 1, 85232 Bratislava

Slovakia

iveta.rizekova@euba.sk 